Document downloaded from:

http://hdl.handle.net/10251/56859

This paper must be cited as:

Lavinia Ricchiuti, A.; Barrera Vilar, D.; Sales Maicas, S.; Thevenaz, L.; Capmany Francoy, J. (2014). Long Weak FBG Sensor Interrogation Using Microwave Photonics Filtering Technique. IEEE Photonics Technology Letters. 26(20):2039-2042. doi:10.1109/LPT.2014.2345611.

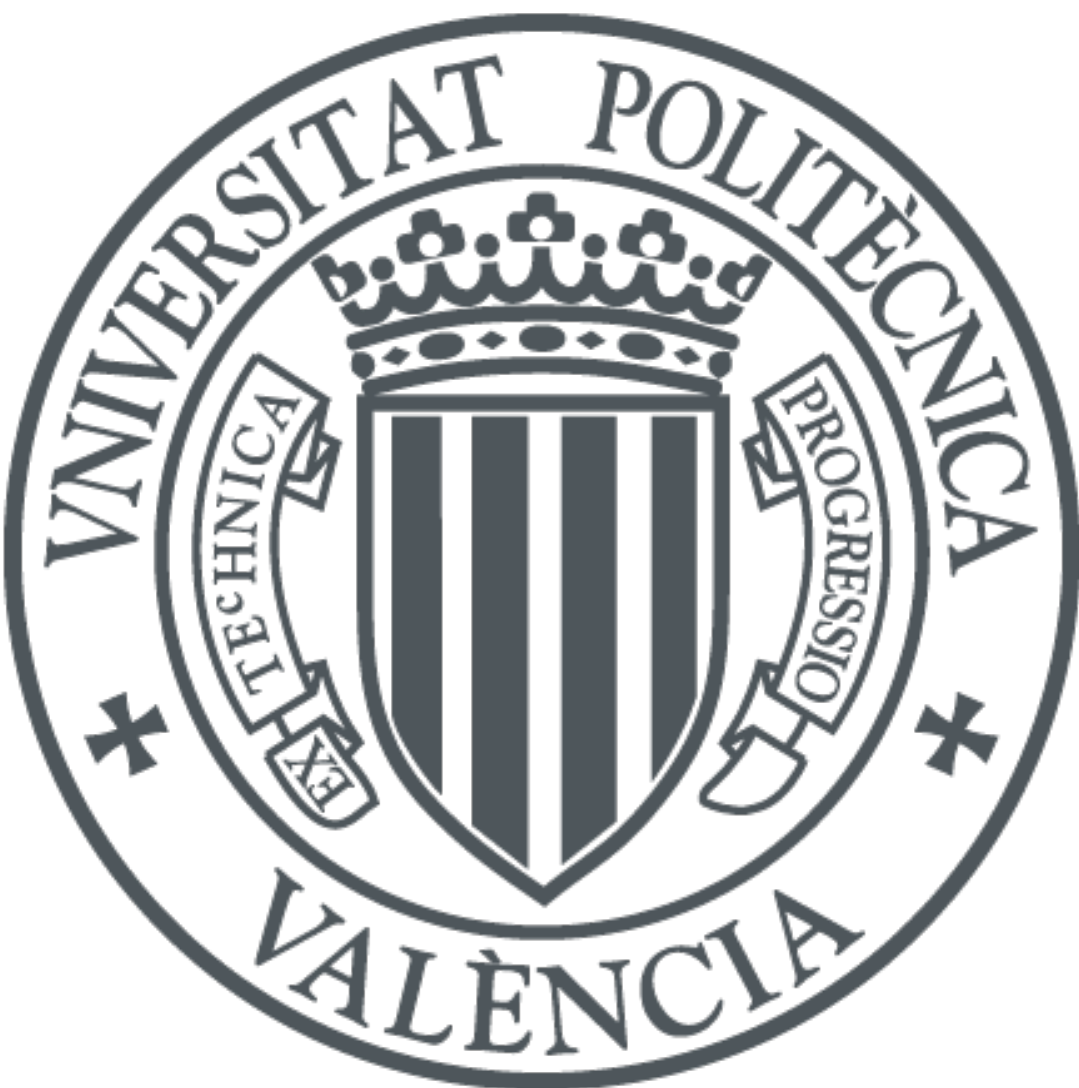

The final publication is available at

http://dx.doi.org/10.1109/LPT.2014.2345611

Copyright Institute of Electrical and Electronics Engineers (IEEE)

Additional Information

"(C) (C) 20xx IEEE. Personal use of this material is permitted. Permission from IEEE must be obtained for all other uses, in any current or future media, including reprinting/republishing this material for advertising or promotional purposes, creating new collective works, for resale or redistribution to servers or lists, or reuse of any copyrighted component of this work in other works." 


\title{
Long Weak FBG Sensor Interrogation using Microwave Photonics Filtering Technique
}

\author{
A.L. Ricchiuti, D. Barrera, S. Sales, L. Thévenaz and J. Capmany
}

\begin{abstract}
A system to interrogate photonic sensors based on long weak fiber Bragg gratings (FBGs) is illustrated and experimentally demonstrated. The FBG sensor is able to detect and measure the precise location of several spot events. The principle of operation is based on a technique used to analyze microwave photonics (MWP) filters. The long weak FBGs are used as quasi-distributed sensors. Several events can be detected along the FBG device with a spatial accuracy of less than $1 \mathrm{~mm}$ using a modulator and a photo-detector (PD) with a modest bandwidth of less than $500 \mathrm{MHz}$. The simple proposed scheme is intrinsically robust against environmental changes and easy to reconfigure.
\end{abstract}

Index Terms-Discrete-time sensors, fiber Bragg gratings, fiber optic sensors, microwave photonics filters, weak FBGs.

\section{INTRODUCTION}

$\mathrm{F}$ BGs have been widely used and have rapidly developed in fiber optic-based signal processing systems and applications due to their excellent properties in terms of simplicity, low insertion loss, polarization independence, low cost and seamless integration in fiber optics systems. In addition, since FBGs are made of dielectric material, they result non conducting, immune to electromagnetic interference (EMI), chemically inert and spark free [1]. These attractive features have made FBGs to be broadly implemented as sensor devices [2]. In this context, several methods have been proposed for interrogating the Bragg-frequency distribution along a FBG with the aim at implementing distributed temperature/strain sensors such as optical low-coherence reflectometry (OLCR) [3], synthesis of optical coherence function (SOCF) [4], frequency analysis [5] and discrete-time microwave photonic filtering technique [6], amongst others.

Manuscript received June 5, 2014; revised July 25, 2014; accepted , 2014 Date of publication , 2014; date of current version July 25, 2014. This work was performed in the framework and with the support of the COST Action TD1001 OFSeSa. The authors acknowledge the Infraestructura FEDER UPVOV08-3E-008, FEDER UPVOV10-3E-492, the Spanish MCINN through the project TEC2011-29120-C05-05, the Valencia Government through the Ayuda Complementaria ACOMP/2013/146, the financial support given by the Research Excellency Award Program GVA PROMETEO 2013/012, and the support from the Swiss Commission for Technology and Innovation (Project 13122.1)

A.L. Ricchiuti, D. Barrera, S. Sales, and J. Capmany are with iTEAM Research Institute, Optical Quantum and Communications Group, Camino de Vera $\mathrm{s} / \mathrm{n}$, Universidad Politécnica de Valencia, 46022 Valencia, Spain (amric1@iteam.upv.es).

L. Thévenaz is with Ecole Polytechnique Fédérale de Lausanne, Institute of Electrical Engineering, SCI-STI-LT Station 11, 1015 Lausanne, Switzerland (luc.thevenaz@epfl.ch).
In this context, OLCR technique has demonstrated very good performance in terms of spatial resolution. Anyway, this scheme presents a limited measurement range and a slow response time. OFDR presents a complex system as it requires post processing and an auxiliary interferometer in order to avoid any non-linearity. Also SOCF demands a very complicated setup and expensive devices.

In this letter, a technique for interrogating long weak FBGs and its potential applications to fiber sensing is described. The proposed configuration is specifically dedicated to the situation when one or more spot events must be precisely detected, such as hot spots or cracks in a structure. This technique offers several advantages since it relies on interference in the microwave domain, which is by far more stable and easier to control than optical domain methods and, if suitably combined with photonics, provides a remarkable spatial accuracy. Furthermore, since the sensor is based on a discrete time filter configuration, the system spectral performance can be tailored/reconfigured. The fundamental concept behind the proposed technique is inspired from the operating principle of a MWP filter [7], in particular on the information on the delays between the different taps of the filter provided by measuring its radiofrequency transfer function given by the $S_{21}$ parameter. In the previous proposed MWP filtering scheme [6], since the FBG has high reflectivity, the most important limitation arises from the fact that the system is not able to detect events having the same magnitude. In this scheme, a FBG sensing device able to detect one or more events having the same magnitude is described. The spot event positions can be evaluated with a spatial accuracy under $1 \mathrm{~mm}$. To demonstrate the performance of the proposed technique two $10 \mathrm{~cm}$-long weak FBGs have been fabricated as a sensing device able to detect one or more spot events located at discrete positions along the grating, using devices with a modest bandwidth of only $500 \mathrm{MHz}$.

\section{DESCRIPTION OF THE METHOD}

The setup to interrogate the long weak FBGs is based on the principle of operation of a MWP filter and is depicted in Fig. 1(a) and Fig. 1(b). A continuous wave (CW) light is electro-optically modulated by a microwave signal. At the output of the electro-optical modulator (EOM) the modulated optical signal is split into $N$ arms. Each arm contains a delayline and an attenuator (or amplifier) in order to provide a delayed and weighted replica of the original signal. These 
time-delayed and weighted optical signals are combined together and photo-detected. In the detection process, the different taps can be mixed under either a coherent or an incoherent basis. In case of incoherent mixing, the tap combination at the PD is insensitive to environmental effects, stable and with a remarkably good repeatability. For these reasons, the experimental setup has been implemented under incoherent operation. The microwave signal is acquired and the electrical frequency response $H(\omega)$ of such a system is given by [7]:

$$
H(\omega)=\sum_{k=0}^{N} a_{k} e^{-i \omega T_{k}}
$$

where $\omega$ is the microwave frequency and $a_{k}$ is the weight of the k-th replica that is delayed by $T_{k}$. If $T_{k}=T, \forall k$ (1) can be identified to a transfer function with a periodic spectral characteristic; the frequency period is known as free spectral range (FSR) and it is inversely proportional to the spacing $T$ between samples [7]. Likewise, the proposed configuration produces delayed replicas of the original signal at the end of the sensing device and at each hot spot to be measured, as illustrated in Fig. 1(b). So, the response of the proposed sensor is described by (1), where the number of taps is equal to the number of hot spots plus one - the reference reflection produced at the end of the weak FBGs sensor. The delay between two consecutive hot-spots $T$ is related to their spacing distance $L$ by [6]:

$$
T=\frac{2 n_{0} L}{c}
$$

where $n_{0}$ is the refractive index of the fiber and $c$ is the speed of light in vacuum.
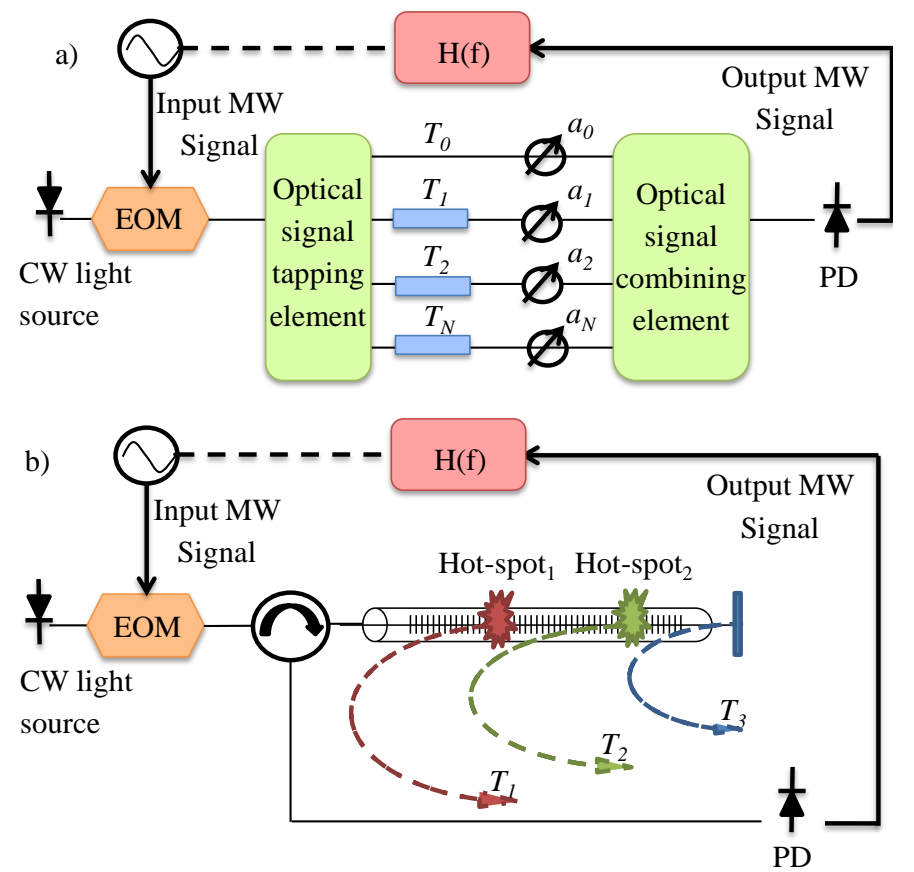

Fig. 1. (a) Scheme of an $N$ tap microwave photonic filter. (b) Schematic diagram of the proposed sensor interrogation.

\section{SETUP AND EXPERIMENTAL MEASUREMENTS}

Experimental measurements have been performed used the setup illustrated in Fig. 2. An optical signal provided by a broad-band source (BBS) is filtered using a tunable band-pass filter centered at the Bragg wavelength of the FBG sensing device, which is $1534.55 \mathrm{~nm}$ at room temperature. The FBGs have a bandwidth of about $16.65 \mathrm{pm}$. As the filter bandwidth is $1 \mathrm{~nm}$, the time coherence of the filtered optical source is 8 ps [8]. The smallest time spacing between hot spots which safely secures the incoherent regime operation would be an order of magnitude bigger than this value. This implies that the distance between spot events should be longer than 8.24 $\mathrm{mm}[8]$.

The output of the tunable filter is electro-optically modulated by a microwave signal generated by a vector network analyzer (VNA), consisting of a radio frequency (RF) tone swept from $10 \mathrm{MHz}$ to $500 \mathrm{MHz}$. At the output of the EOM, the signal is sent into the FBG sensor through an optical circulator. The frequency response of the system is analyzed by monitoring the scattering parameter $S_{21}$, which relates the detected signal to the input modulating microwave signal.

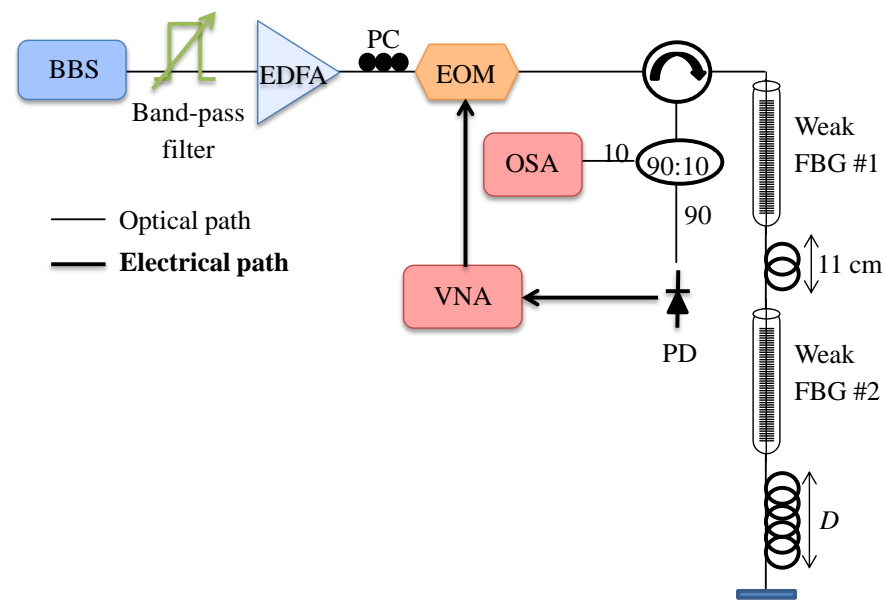

Fig. 2. Setup used for interrogating a quasi-distributed weak FGBs sensor.

The initial idea of this experiment was to fabricate a very long weak FBG, but due to the limitations of our FBGs fabrication system, FBGs longer than $10 \mathrm{~cm}$ cannot actually be fabricated. Hence, as a proof-of-concept, the quasidistributed sensor proposed is made by a pair of weak $(R<6 \%)$ $10 \mathrm{~cm}$-long FBGs separated by $11 \mathrm{~cm}$, while a piece of singlemode fiber (SMF) of length $D=7.45 \mathrm{~m}$ is appended after the second FBG. The other end of this fiber is left opened in the air to provide a reflection signal that will be used as reference tap.

If a $5 \mathrm{~mm}$ hot spot is placed at a certain point of the first grating, this will produce a local Bragg frequency shift and generate a reflected signal at the point where the hot spot is placed. The wavelength sweep of the optical band-pass filter is synchronized with the data acquisition process. This way, by performing a wavelength scan of the input signal, the amplitude of the MWP filter response can be maximized. Hence, by tuning the optical band-pass filter, the taps related to the original FBGs reflections are filtered out and a two tap 
MWP filter is actually obtained (see Fig. 3(a)) - resulting from the combination of the tap provided by the signal reflected by the hot spot and the reference tap.
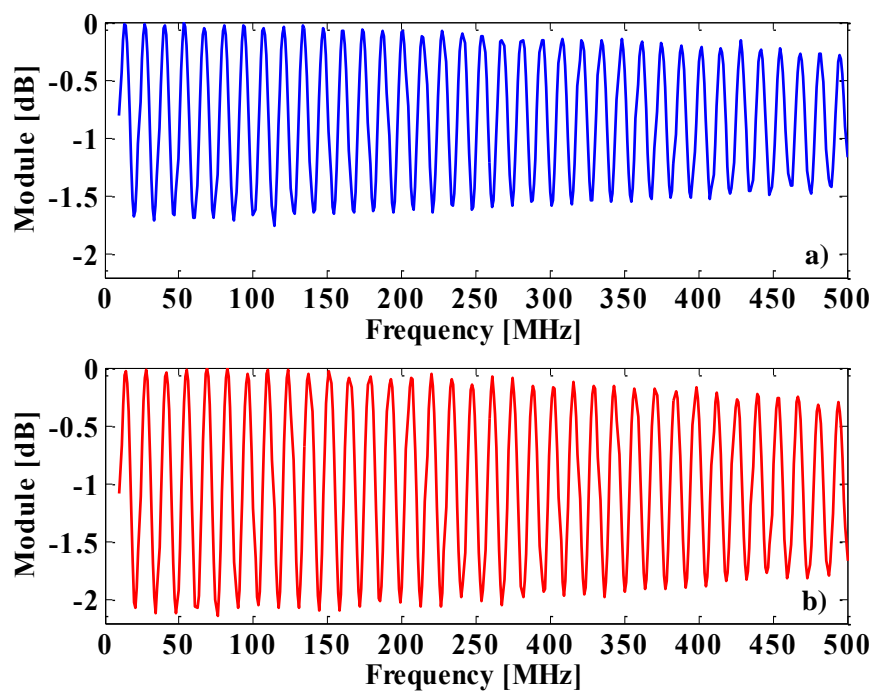

Fig. 3. (a) MWP two tap filter created by the reference tap and the tap provided by the hot spot placed in the first FBG. (b) MWP two tap filter obtained by the reference tap and the tap provided by the hot spot placed in the second FBG.

In the same way, if the hot spot is removed from the first FBG and placed along the second grating, another two tap filter is created, as shown in Fig. 3(b). The locations of the spot events can be obtained directly by evaluating the FSR of the filters as it is described by (2), or by using the inverse Fourier transform (IFT). The FSR has been evaluated with a frequency step of $1 \mathrm{kHz}$, which corresponds to an estimated spatial accuracy of less than $1 \mathrm{~mm}$ [6]. This way, the distances between the end of the SMF and the two hot spots placed in the first and the second FBG are $l_{1}=7.7162 \mathrm{~m}$ and $l_{2}=7.5062$ $\mathrm{m}$, respectively.

When two hot spots are placed along each FBG and the band-pass filter is properly tuned in order to eliminate the original FBG reflections, a three tap filter is obtained, as shown in Fig. 4(a). In this case, the most efficient approach to calculate the positions of the two hot spots referred to the end of the SMF is simply to take the IFT [6] of the measured $S_{21}$ parameter, which is depicted in the inset of Fig. 4(a). The above-mentioned distance are calculated to be $l_{l}=7.7166 \mathrm{~m}$ and $l_{2}=7.5063 \mathrm{~m}$, in agreement with the values estimated previously. Furthermore, the distance between the two hot spots, $L_{l}$, can be measured evaluating the FSR of the two tap filter, obtained if the reflection at the end of SMF is suppressed, as illustrated by the blue curve in Fig. 4(b). In order to eliminate this reference tap, an angled polished connector (APC) and an index-matching fluid (IMF) are now applied at the end of the SMF. This way, if the second hot spot is moved, the FSR of the two tap filter changes, as shown by the red curve in Fig. 4(b). Once the FSR calculated, the distance between spot events can be evaluated by (2).
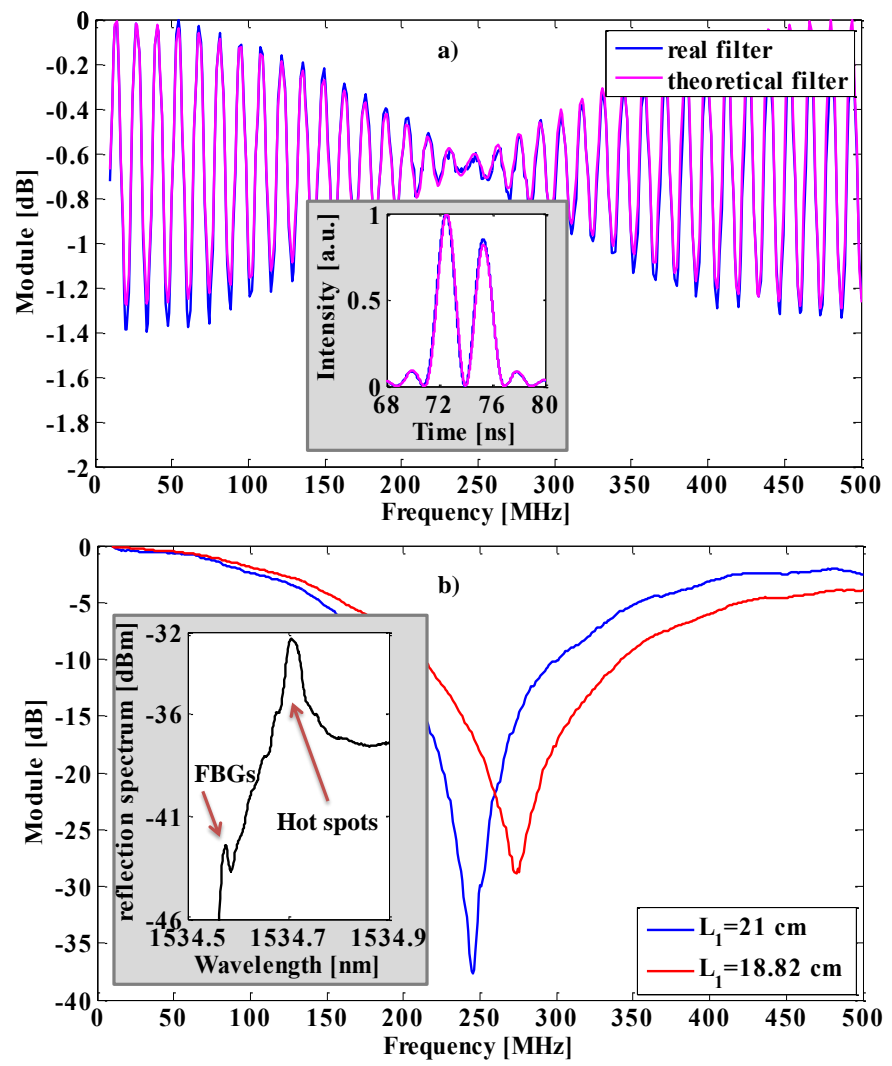

Fig. 4. (a) Frequency response of the three tap filters (real and theoretical) obtained by placing two hot spots along the sensor and using the end of the fiber as reference tap. Inset: IFT of the three tap filters depicted in Fig. 4(a). (b) Frequency response of the two taps filters obtained by placing two hot spots along the sensor and eliminating the reference tap. Inset: Reflection spectrum acquired with the OSA.

Moreover, in order to estimate the temperature of the hot spots an optical spectrum analyzer (OSA) is used (see Fig. 2). Since a $10 \mathrm{pm}$ shift in Bragg wavelength corresponds to $1{ }^{\circ} \mathrm{C}$ shift in temperature, the temperature shift in the hot spot zone is calculated to be $\Delta \mathrm{T}=16^{\circ} \mathrm{C}$ (see the inset of Fig. 4(b)).

Finally, it is worth mentioning that since the filter formed by the taps provided by the hot spots has just one FSR span (see Fig. 4(b)), the minimum distance between two events should be more than $20 \mathrm{~cm}$ if an accuracy under $1 \mathrm{~mm}$ is desirable. Actually, this value arises from the modest bandwidth used to perform the experiment which means that the minimum distance between events could be further reduced by extending the frequency range. In fact, in order to correctly estimate the distance between hot spots by means of the IFT, the frequency response of the related MWP filter has to present at least one FSR span, if an accuracy of less than $1 \mathrm{~mm}$ is desirable. For this reason, the minimum detectable distance between spot events $L_{\min }$ is directly related to the VNA frequency span $\Delta f_{V N A}$, according to:

$$
L_{\min }=\frac{c}{2 n_{0} \Delta f_{V N A}}
$$

By using (3) the necessary increase in frequency span to reduce the distance between spot events to a desired value can be estimated. 


\section{CONCLUSIONS}

A technique for estimating the position and number of spot events along a weak FBG-based sensor is proposed and demonstrated, based on the principle of operation of a MWP filter. The hot spot positions can be simply evaluated by using an EOM and a PD of only $500 \mathrm{MHz}$ bandwidth, while the temperature of the hot spots can be measured by means of an OSA. The initial idea of this experiment was to fabricate a very long weak FBG, but due to the limitations of our grating fabrication system, FBGs longer than $10 \mathrm{~cm}$ cannot be created yet. Hence, as a proof-of-concept a pair of weak $10 \mathrm{~cm}$-long FBGs is employed as a quasi-distributed temperature/crack sensor. Notwithstanding this artefact, the sensing range could be further improved by fabricating a longer FBG-based sensor and implementing the presented methodology. By evaluating the FSR of the resulting MWP filter the location of two hot spots along the FBG sensor can be detected with a remarkable accuracy under $1 \mathrm{~mm}$. This value can be further improved using a higher range instrument.

Moreover, relying on microwave interferometry and working under incoherent operation, the configuration proposed results intrinsically robust against environmental changes, stable and with remarkable good repeatable performance. For these reasons the measurements of the MWP filter response have been achieved with no need of averaging mode.

The proposed technique is potentially quasi-cost effectively solution as it is based on low bandwidth RF and off-the-shelf photonic components rather than on ultra-short pulses, optical interferometry or OFDR techniques. Furthermore, the instrumentation required could be further simplify by replacing the VNA with a tunable oscillator and a device able to analyze the magnitude response of the MWP filter generated up to $500 \mathrm{MHz}$.

The proposed configuration allows the detection and estimation of several spot events by using the IFT of the measured RF transfer function $\left(S_{21}\right.$ parameter). The minimum detectable distance between two events has to be more than 20 $\mathrm{cm}$, dictates by the frequency span used. Even so, it is possible to release this limitation if the number of replicas of the filter is increased, either by extending the frequency range or by mathematically improving the algorithm used to calculate the IFT. The latter point is actually a complementary research under study going beyond the scope of this manuscript.

\section{REFERENCES}

[1] B. Culshaw, "Optical fiber sensor technologies: opportunities and perhaps pitfalls," J. Lightw. Technol., vol. 22, pp. 39-50, Jan. 2004.

[2] A. D. Kersey et al., "Fiber grating sensors," J. Lightw. Technol., vol. 15, no. 8, pp. 1442-1463, Aug. 1997.

[3] M. Volanthen, H. Geiger, and J. P. Dakin., "Distributed grating sensors using low-coherence reflectometry," J. Lightw. Technol., vol. 15, no. 11, pp. 2076-2082, Nov. 1997.

[4] K. Hotate and K. Kajiwara, "Proposal and experimental verification of Bragg wavelength distribution measurement within a long-length FBG by synthesis of optical coherence function," Opt. Express, vol. 16, no. 11, pp. 7881-7887, May 2008.

[5] J. Sancho et al., "Time-frequency analysis of long fiber Bragg gratings with low reflectivity", Opt. Express, vol. 21, no. 6, pp. 7171-7179, Mar. 2013.
[6] A. L. Ricchiuti et al., "Long fiber Bragg grating sensor interrogation using discrete-time microwave photonic filtering techniques," Opt. Express, vol. 21, no. 23, pp. 28175-28181, Nov. 2013.

[7] J. Capmany et al., "Microwave photonic signal processing," J. Lightw. Technol., vol. 31, no. 4, pp. 571-586, Aug. 2013.

[8] B. E. A. Saleh and M. C. Teich, Fundamentals of Photonics, 2nd ed., New York, Wiley, 2007. 